\title{
NATURE OF THE MHD AND KINETIC SCALE TURBULENCE IN THE MAGNETOSHEATH OF SATURN: CASSINI OBSERVATIONS
}

\author{
L. Z. Hadid ${ }^{1}$, F. Sahraoui ${ }^{1}$, K. H. Kiyani ${ }^{1,2}$, A. Retinò ${ }^{1}$, R. Modolo ${ }^{3}$, P. Canu ${ }^{1}$, A. Masters ${ }^{4}$, And M. K. Dougherty ${ }^{5}$ \\ ${ }^{1}$ Laboratoire de Physique des Plasmas, CNRS-Ecole Polytechnique-UPMC, Observatoire de Saint-Maur, France; lina.hadid@lpp.polytechnique.fr \\ ${ }^{2}$ Centre for Fusion, Space and Astrophysics, University of Warwick, Coventry CV4 7AL, UK \\ ${ }^{3}$ LATMOS, CNRS-UVSQ-UPMCS, Guyancourt, France \\ ${ }^{4}$ ISAS-JAXA, Sagamihara, Japan \\ ${ }^{5}$ The Blackett Laboratory, Imperial College London, London SW7 2BZ, UK \\ Received 2015 August 18; accepted 2015 October 11; published 2015 November 4
}

\begin{abstract}
Low-frequency turbulence in Saturn's magnetosheath is investigated using in situ measurements of the Cassini spacecraft. Focus is put on the magnetic energy spectra computed in the frequency range of $\sim\left[10^{-4}, 1\right] \mathrm{Hz}$. A set of 42 time intervals in the magnetosheath were analyzed, and three main results that contrast with known features of solar wind turbulence are reported. (1) The magnetic energy spectra showed a $\sim f^{-1}$ scaling at MHD scales followed by an $\sim f^{-2.6}$ scaling at sub-ion scales without forming the so-called inertial range. (2) The magnetic compressibility and the cross-correlation between the parallel component of the magnetic field and density fluctuations $C\left(\delta n, \delta B_{||}\right)$indicate the dominance of the compressible magnetosonic slow-like modes at MHD scales rather than the Alfvén mode. (3) Higher-order statistics revealed a monofractal (multifractal) behavior of the turbulent flow downstream of a quasi-perpendicular (quasi-parallel) shock at sub-ion scales. Implications of these results on theoretical modeling of space plasma turbulence are discussed.
\end{abstract}

Key words: magnetic fields - planets and satellites: individual (Saturn) - plasmas - turbulence - waves

\section{INTRODUCTION}

The solar wind is unmatched by any other astrophysical system at the level of detail in which turbulence can be investigated. This is due the availability of many spacecraft missions that provide high-quality field and particle in situ measurements. The available data allowed significant progress in understanding turbulence and energy dissipation in collisionless magnetized plasmas. One of the most common and insightful ways of measuring the multiscale nature of turbulence is via the power spectral density (PSD) of the turbulent fluctuations. From that point of view, it has been shown that the magnetic energy spectrum in the solar wind is generally characterized by at least four different dynamical ranges of scales. First is the energy-containing range that follows a scaling of $\sim f^{-1}$, which is observed essentially in the fast solar wind and thought to be filled by uncorrelated randomlike fluctuations that may originate from reflected waves in the solar corona (Bavassano et al. 1982; Velli et al. 1989). The second region is the so-called inertial range with a scaling of $\sim f^{-5 / 3}$ or $\sim f^{-3 / 2}$ thought to originate from nonlinear interactions between counter-propagating incompressible Alfvénic wave-packets transferring the energy down to shorter wavelengths (Iroshnikov 1964; Kraichnan 1965). This spectrum terminates with a breakpoint occurring near the ion gyroscale or inertial length scale that is generally followed by a steeper power-law spectrum $f^{-\alpha}$ at sub-ion scales with a broader range of slopes, $\alpha \in[-2.3,-3.1]$, where the magnetic energy starts to dissipate into particle heating (Goldstein et al. 1994; Leamon et al. 1998a; Alexandrova et al. 2008; Hamilton et al. 2008; Kiyani et al. 2009b; Sahraoui et al. 2009). As the cascade approaches the electron scale, the spectrum steepens again, which is interpreted as being due to dissipation of the remaining magnetic energy into electron heating via Landau damping of kinetic Alfvén wave (KAW) turbulence (Leamon et al. 1998b, 1999; Hollweg 1999; Howes et al. 2006; Sahraoui et al. 2009). Due to instrumental limitations, the actual scaling at sub-electron scales and the fate of the energy cascade remain open questions (see the discussions in Sahraoui et al. 2013).

Turbulence in the terrestrial magnetosheath is more complex than in the solar wind as different waves and instabilities can be generated by, e.g., temperature anisotropy generally observed behind the bow shock. Moreover, boundaries such as the magnetopause and the shock may influence some of the turbulence properties (e.g., its spatial anisotropy; Russell et al. 1990; Cattaneo et al. 2000; Sahraoui et al. 2006; Yordanova et al. 2008). Previous studies of magnetic energy spectra in the terrestrial magnetosheath showed some similarities with the solar wind: the presence of the Kolmogorov spectral index $-5 / 3$ at MHD scales (Sundkvist et al. 2007; Alexandrova et al. 2008) and a broad range of slopes, [-2.5, -3], at sub-ion scales (Czaykowska et al. 2001; Huang et al. 2014). Some differences seem to exist regarding the scaling at sub-electron scales (Huang et al. 2014).

In planetary systems other than Earth, turbulence is much less explored. For turbulence studies, there is at least one major point of interest in investigating planetary magnetospheres: they offer access to a broader range of plasma parameters that are not available in near-Earth space (von Papen et al. 2014; Tao et al. 2015). This is the case, for example, of the Alfvén Mach number, relevant for the physics of shocks and compressible turbulence, which can reach values as high as $\sim 100$ near Saturn (Masters 2013). The reason for this is that the magnetic field magnitude and the density fluctuations decrease with different scaling laws, whereas the solar wind speed stays relatively constant (Masters et al. 2011). Another point of interest is understanding the role that the planet's satellites (e.g., Io for Jupiter) may play in modifying locally the turbulence properties through different plasma processes and instabilities that the planet-moon coupling may generate (Kivelson et al. 2004; Saur et al. 2004; Chust et al. 2005; 

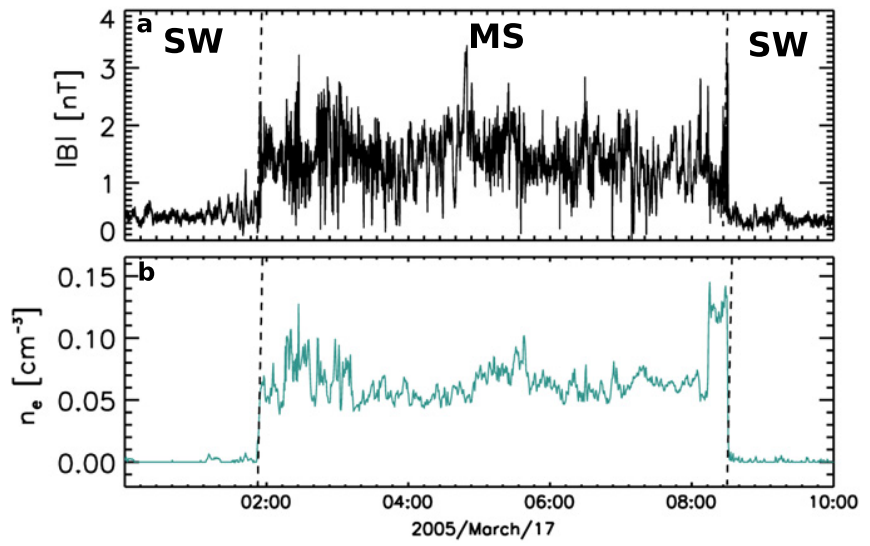

Figure 1. (a) Magnetic field modulus and (b) electron plasma density measured by the Cassini spacecraft in the solar wind (SW) and in the magnetosheath (MS) of Saturn on 2005 March 17 from 00:00 to 10:00.

Bagenal 2007). Using a list of long (several hours) crossings of the Kronian magnetosheath by the Cassini spacecraft, we investigate the properties of turbulence at MHD and sub-ion (kinetic) scales and compare them to the previously reported ones in the solar wind and in the terrestrial magnetosheath. We try to answer three main questions regarding turbulence in the magnetosheath. Is the $f^{-5 / 3}$ Kolmogorov inertial range ubiquitous? What is the nature of the plasma mode(s) (e.g., Alfvénic or magnetosonic) that dominate the cascade at different scales? Do turbulence properties depend on the local plasma parameters (e.g., the normal angle to the shock)?

\section{RESULTS}

\subsection{Statistics of the Spectral Slopes}

Figure 1 illustrates a typical magnetosheath crossing on 2005 March 17 at 02:00 UT and 08:30 UT (7.30 and 7.35 LT, respectively) at a distance of $\sim 42 R_{s}\left(1 R_{s}=60.268 \mathrm{~km}\right)$. From the field magnitude and the density measurements, we see that the spacecraft was in the solar wind until about 02:00 UT, when it encountered the bow shock and entered into the magnetosheath where the field strength and the density increased significantly. The magnetic field data, sampled at $32 \mathrm{~Hz}$, were measured by the Flux Gate Magnetometer (FGM) sensor from the Cassini MAG experiment (Dougherty et al. 2004). The FGM is mounted halfway along the $11 \mathrm{~m}$ spacecraft boom to minimize the interference from the spacecraft-generated electromagnetic fields. The ion and electron moments were measured by the Cassini Plasma Spectrometer (CAPS; Young et al. 2004). Cassini being a nonspinning spacecraft and the CAPS sensor having a limited field of view (FOV), a careful handling of the thermal ion population is required because the ion thermal speed is smaller than the bulk flow speed. In fact, the moments are not reliable when the bulk of the plasma flow is not in the FOV of the ion instrument (Thomsen et al. 2010; Romanelli 2014). However, since the electrons have a thermal speed that is larger than the bulk flow speed downstream of the bow shock, the previous condition can be relaxed, and the electron can be assumed to be isotropic (at least on the large timescales that we consider in this work; Lewis et al. 2008). This implies that electron moments, computed from the ELS instrument, would have fewer uncertainties than the ion ones. For that reason, we use the electron density measured by ELS as the plasma density under

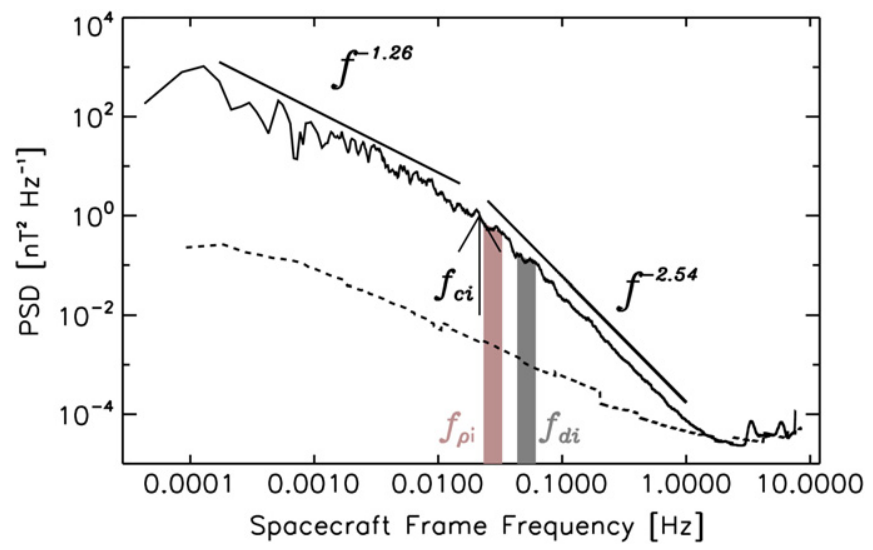

Figure 2. Power spectral density of $\delta B$ measured between 02:00 and 08:30. The black lines are the power-law fits. The dotted curve is a spectrum measured in the solar wind, considered here to represent the upper bound of the sensitivity floor of FGM. The arrow corresponds to the ion gyrofrequency; the gray and the red shaded bands indicate the Taylor-shifted ion inertial length $f_{d i}$ and Larmor radius $f_{\rho_{i}}$, respectively (the width reflects the uncertainty due to errors in estimating the ion moments).

the assumption of quasi-neutrality, $n_{i} \sim n_{e} \sim n$ (Figure 1(b)). Figure 2 shows the PSD of the magnetic field fluctuations computed using a windowed fast Fourier transform (FFT). The spectrum shows two ranges of scales with distinct power laws: $\sim f^{-1.26}$ at low frequencies $\left(f<10^{-2} \mathrm{~Hz}\right)$ and $\sim f^{-2.54}$ at higher frequencies. This observation shows a striking result: turbulence transits directly from the "energy-containing scales" into the ion kinetic scales, without forming the so-called Kolmogorov inertial range with a scaling of 5/3 (the terminology of energy-containing scales is borrowed from solar wind turbulence). The spectral break is closer to the local ion gyrofrequency than to the Taylor-shifted ion inertial length $f_{d_{i}}=V_{f} / 2 \pi d_{i}$ and Larmor radius $f_{\rho_{i}}=V_{f} / 2 \pi \rho_{i} \quad\left(V_{f} \sim\right.$ $300 \mathrm{~km} \mathrm{~s}^{-1}$ is the average flow speed, $T_{i} \sim 258 \mathrm{eV}, B_{0} \sim$ $1.4 \mathrm{nT}, n_{e} \sim 0.06 \mathrm{~cm}^{-3}$, and $\beta_{i} \sim 3.3$ ). The reason might be that the latter are subject to higher uncertainties due to errors in estimating the plasma parameters using the ion moments from the CAPS instrument. To confirm the absence of the Kolmogorov $f^{-5 / 3}$ spectrum in the magnetosheath, we analyzed a list of 42 other time intervals between 2004 and 2007 for quasi-parallel and quasi-perpendicular shocks separately. For most of the time intervals, we identified the structure of the shock by checking the angle $\theta_{B n}$ between the interplanetary magnetic field and the normal to the shock estimated using a semi-empirical model of the global shock surface (Went et al. 2011): $\theta_{B n}>45^{\circ}$ indicates a quasi-perpendicular shock, whereas $\theta_{B n} \leqslant 45^{\circ}$ indicates a quasi-parallel one (in a few cases, quasi-perpendicular shocks are simply identified by a sharp gradient in the magnetic field and the plasma measurements). The results shown in Figure 3 statistically confirm the absence of the Kolmogorov spectrum at MHD scales: the bulk of the spectra had slopes near -1 for quasi-perpendicular shocks in particular (Czaykowska et al. 2001). The histogram of the slopes at sub-ion scales peaks between $[-2.5,-3]$, in general agreement with previous results reported in the solar wind and the magnetosheath (Sahraoui et al. 2013; Huang et al. 2014). A slight indication that steeper spectra are observed behind quasi-parallel shocks can also be seen. 

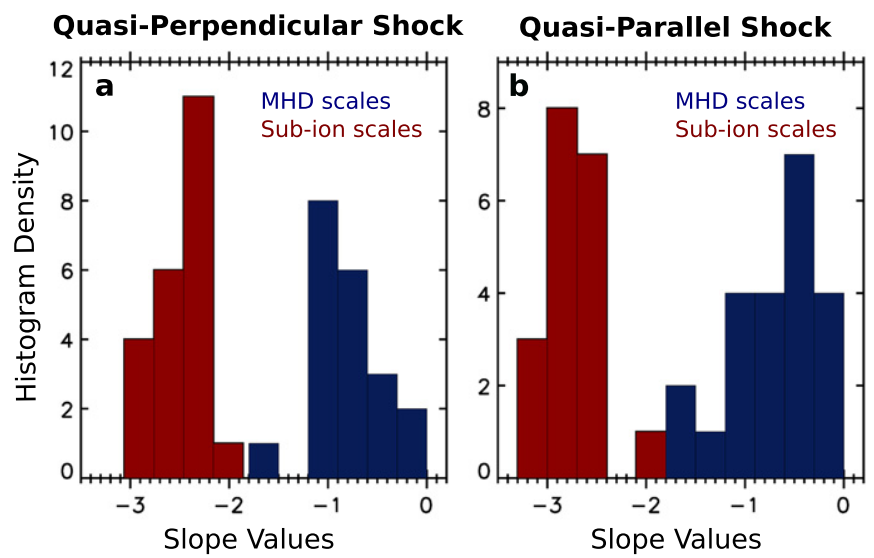

Figure 3. Histograms of the spectral slopes at MHD and sub-ion (kinetic) scales downstream of (a) quasi-perpendicular and (b) quasi-parallel shocks.

\subsection{Nature of the Turbulent Fluctuations at the MHD and Kinetic Scales}

To identify the nature of plasma modes that carry the energy cascade from the energy-containing to the sub-ion scales, we use the magnetic compressibility $C_{B}$ given by the ratio between the PSDs of the parallel magnetic field component and the magnetic field magnitude (parallel with respect to the background magnetic field $B_{0}$; Gary \& Smith 2009; Salem et al. 2012):

$$
C_{B}(f)=\frac{\left|\delta B_{\|}(f)\right|^{2}}{\left|\delta B_{\|}(f)\right|^{2}+\left|\delta B_{\perp}(f)\right|^{2}}
$$

Indeed, from linear theory, the Alfvén and the magnetosonic modes are known to have very different profiles of magnetic compressibility (Sahraoui et al. 2012). This allows us to verify easily the dominance (or not) of the Alfvénic fluctuations in our data (Podesta \& TenBarge 2012; Kiyani et al. 2013). We computed the theoretical magnetic compressibilities from the linear solutions of compressible Hall-MHD (Sahraoui et al. 2003) and from the Maxwell-Vlasov equations using the WHAMP code (Rönnmark 1982). For the sake of simplicity, we keep using the terminology of the MHD slow and fast modes at kinetic scales, even if it may be inadequate (because of possible crossings between different dispersion branches). Since the slow mode is heavily damped in kinetic theory at finite $\beta_{i}$ (Ito et al. 2004; Howes 2009), we used the limit $\beta_{i}=0$ and $\beta_{e}=1$ (therefore, $\beta=\beta_{e}+\beta_{i}=1$ ) to suppress the ion Landau damping and thus to capture the slow mode solution down to the scale $k \rho_{i} \geqslant 1$. In order to compare to spacecraft observations, knowledge of the three components of the $\boldsymbol{k}$ vector (or, equivalently, the propagation angle $\Theta_{k B_{o}}$ and the modulus $k$ ) from the data is required. However, unambiguous determination of those quantities requires having multispacecraft data that is not available in planetary magnetospheres other than Earth (Sahraoui et al. 2006). Therefore, we use the Taylor frozen-in-flow hypothesis, which assumes that the fluctuations have slow phase speeds with respect to the flow speed, to infer the component of $\boldsymbol{k}$ along the flow direction, i.e., $\omega_{s c} \sim \boldsymbol{k} . \boldsymbol{V}_{f} \sim k V_{f}$. Under the assumption that turbulence is strongly anisotropic, i.e., $k_{\perp} \gg k_{\|}$, which is supported by

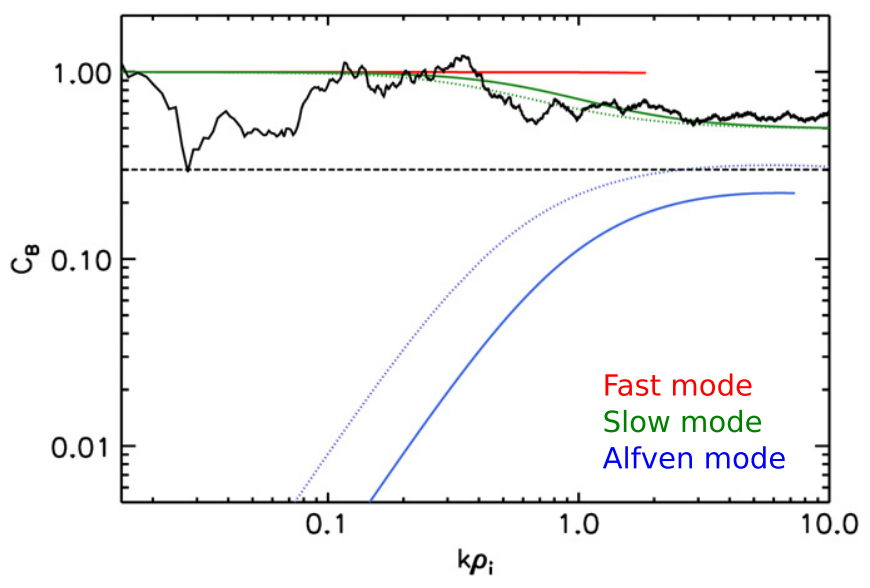

Figure 4. Comparison between theoretical magnetic compressibilities, computed from the linear solutions of the compressible Hall-MHD (colored dotted lines) and of the Vlasov-Maxwell equations (colored solid lines) for $\beta=1$ and $\Theta_{k B}=87^{\circ}$, with the observed one from the data of Figure 1 (02:00-08:30; solid black curve). The Taylor hypothesis was used to convert the frequencies in the spacecraft frame into wavenumbers. The red, green, and blue curves correspond respectively to the theoretical fast, slow, and KAW modes. The horizontal dashed black line at $C_{B}=1 / 3$ indicates the power isotropy level.

previous observations in the magnetosheath (Mangeney et al. 2006; Sahraoui et al. 2006) and in the solar wind (Sahraoui et al. 2010), the estimated wavenumber component along the flow is equivalent to $k_{\perp}$ for data intervals when $\Theta_{V_{f} \boldsymbol{B}_{0}} \sim 90^{\circ}$. In the present data we estimated $\Theta_{V_{f} B_{0}} \sim 87^{\circ}$, which we used to compute the theoretical solutions of Figure 4 assuming $\Theta_{V_{f} \boldsymbol{B}_{0}} \sim \Theta_{k B_{0}}$. Nevertheless, we performed a parametric study (not shown here) and verified that the magnetic compressibilities of the compressible Hall-MHD solutions keep the same profile (but change its magnitude) when varying $\beta$ in the range $[0.2,100]$ for a fixed $\Theta_{k B_{o}}=87^{\circ}$ and when varying $\Theta_{k B_{o}}$ from quasi-parallel to quasi-perpendicular angles for $\beta=1$. Another consequence of using the Taylor hypothesis when $\Theta_{V_{f} B_{0}} \sim 90^{\circ}$ is that the perpendicular component of the fluctuation $\delta B_{\perp}$ in Equation (2) is reduced to the component perpendicular to both $\boldsymbol{V}_{f} \quad$ (or $\boldsymbol{k}$, to fulfill $\boldsymbol{k} . \boldsymbol{\delta} \boldsymbol{B}=0$ ) and $\boldsymbol{B}_{0}$ (Podesta \& TenBarge 2012), namely,

$$
\delta B_{\perp}(f)=\boldsymbol{\delta} \boldsymbol{B}(f) . \frac{\boldsymbol{k} \times \boldsymbol{B}_{0}}{\left|\boldsymbol{k} \times \boldsymbol{B}_{0}\right|} \sim \boldsymbol{\delta} \boldsymbol{B}(f) \cdot \frac{\boldsymbol{V}_{f} \times \boldsymbol{B}_{0}}{\left|\boldsymbol{V}_{f} \times \boldsymbol{B}_{0}\right|} .
$$

Figure 4 shows a comparison between the observed magnetic compressibilities (from the data of Figure 1) compared to theoretical ones calculated using the observed plasma parameters. First, one can see that the theoretical magnetic compressibilities of the fast and slow modes in the fluid and kinetic models have the same profile being almost constant at the MHD and sub-ions scales. The KAW mode shows an increasing magnetic compressibility as it approaches kinetic scales (Sahraoui et al. 2012). A similar profile has been reported in solar wind observations (Podesta \& TenBarge 2012; Kiyani et al. 2013). Second, the measured magnetic compressibility shows a relatively constant and high level $\left(C_{B}>1 / 3\right)$ at the energy-containing scales and in the sub-ions range, which indicates the dominance of the parallel component $\delta B_{\|}$(most of the 42 studied intervals showed a similar profile). This clearly 


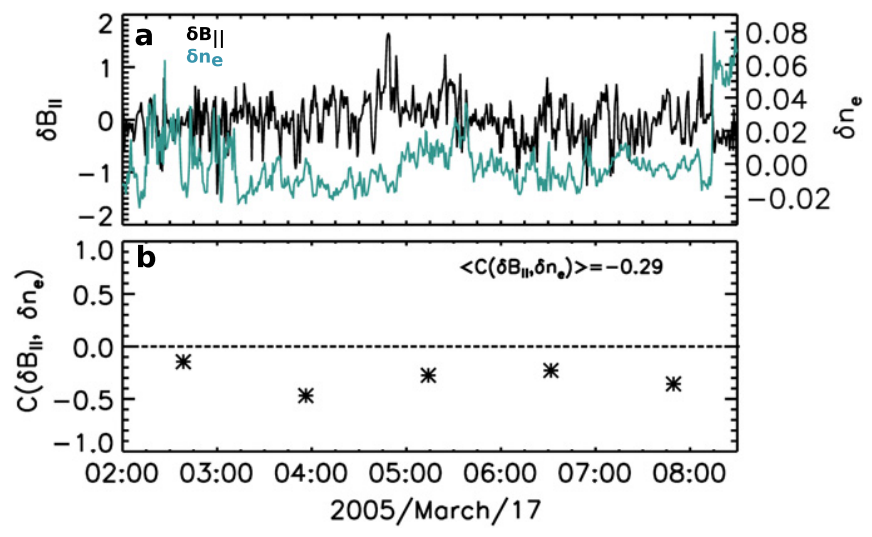

Figure 5. (a) Plasma density and the magnetic field magnitude. (b) Local and averaged cross-correlation of the density and the parallel component of the magnetic fluctuations calculated using Pearson's method.

rules out the Alfvénic fluctuations as a dominant component of the turbulence, at least at MHD scales $(f<0.05 \mathrm{~Hz})$.

Figure 4 shows that the magnetic compressibility cannot be used to distinguish between fast and slow modes. To do so, we use instead the cross-correlation between the magnetic field and the plasma density fluctuations $C\left(\delta B_{\|}, \delta n\right)$. Indeed, the fast (slow) mode is known to have a correlation (anti-correlation) between its density and parallel magnetic component (Gary \& Winske 1992). Figure 5 shows that locally and on average the density and the parallel component of the magnetic fluctuations are anti-correlated, i.e., $C\left(\delta B_{\|}, \delta n_{e}\right)<0$. This clearly rules out the fast mode fluctuation as the dominant component of the turbulence. This analysis establishes that the magnetosonic slow-like mode dominates the turbulent fluctuations analyzed here in agreement with previous results on the Earth's magnetosheath (Kaufmann et al. 1970; Song et al. 1994; Cattaneo et al. 2000), in outer planets (Violante et al. 1995; Erds \& Balogh 1996), and in the solar wind (Howes et al. 2012; Klein et al. 2012). However, one cannot rule out the possible presence of the ion mirror mode as previously reported in the terrestrial magnetosheath (Sahraoui et al. 2006). The mirror mode, although of a purely kinetic nature (Southwood \& Kivelson 1993), indeed has very similar properties to the slow mode, which makes it challenging to distinguish between the two modes in the spacecraft data. To check the possibility of the mirror mode existing in our data requires measuring at least the ion temperature anisotropy, which is not available on board the Cassini spacecraft.

\subsection{Higher-order Statistics of the Magnetic Field Fluctuations}

To investigate the monofractal versus multifractal nature of the observed turbulence, we analyzed the probability density function (PDF) of the magnetic field temporal increments, defined as $\delta B_{\tau}(t, \tau)=B(t+\tau)-B(t)$, where $\tau$ is the time lag. Intermittency is generally characterized by the presence of bursty increments that yield heavy tails in the PDF of the field increments at small scales. In general, it is this deviation from Gaussianity that contains the pertinent information about the underlying physics. Figure 6 provides three examples of the corresponding PDFs obtained from the list of the analyzed events downstream of quasi-parallel $\left(\Theta=31^{\circ}\right)$ and quasiperpendicular shocks $\left(\Theta=86^{\circ}\right.$ and $\Theta=60^{\circ}$, respectively) for two values of $\tau$, one from the MHD range $(\tau \sim 975 \mathrm{~s})$ and one from the sub-ion range $(\tau \sim 25 \mathrm{~s})$. Figures 6(a)-(b) show that behind the quasi-perpendicular shock, the PDFs are found to be quasi-Gaussian for both values of $\tau$ (i.e., at MHD and kinetic scales) indicating the quasi-randomness of the fluctuations in the "energy-containing scales" and in the sub-ion range. On the contrary, behind the quasi-parallel shock (Figure 6(c)), the PDFs are non-Gaussian for $\tau \sim 25 \mathrm{~s}$ (red PDF) showing the intermittent nature of turbulence at the kinetic scales as it was observed in the terrestrial magnetosheath (Sundkvist et al. 2007; Yordanova et al. 2008). In the energy-containing scales, the PDF is quasi-Gaussian as in the case of a quasiperpendicular shock. These results agree with recent findings using global hybrid simulations (Karimabadi et al. 2014). Next, we calculate higher-order statistics given by the structure functions (SFs) of the magnetic field increments defined in Equation (3).

$$
S_{m}(\tau)=\int_{-\infty}^{\infty}\left|\delta B_{\tau}(t)\right|^{m} P(\delta B) d t=\left\langle\left|\delta B_{\tau}(t)\right|^{m}\right\rangle .
$$

When increasing the order $m$, the SFs become progressively sensitive to rare and bursty events. Assuming a power-law dependence of the SFs as function of $\tau$, i.e., $S_{m}(\tau) \sim \tau^{-\zeta(m)}$, a linear (nonlinear) dependence of $\zeta(m)$ on the order $m$ indicates a monofracatal (multifractal) behavior of the turbulence. The scaling exponent in Figure 7(a) shows a clear linear dependence of $\zeta$ on $m$ at the sub-ion scales (三 small values of $\tau$ ) behind a quasi-perpendicular shock thus supporting the monofractal character of the turbulent fluctuations.

However, downstream of a quasi-parallel shock (Figure 7(c)), the scaling exponent is a convex function of $m$, confirming the multifractal nature of turbulence at the kinetic scales. We recall that in the solar wind it has been shown that the sub-ion scales were monofractal while the MHD scales were multifractal (Kiyani et al. 2009b).

\section{DISCUSSION AND CONCLUSIONS}

A benefit of analyzing sufficiently long and relatively stationary time series measured by the Cassini spacecraft in the magnetosheath of Saturn was that we were able to probe into more than four decades of scales spanning from the MHD down to sub-ion scales. We present the following plausible, albeit speculative, scenario to explain the different observations: the interaction of the solar wind with the bow shock may lead to the destruction of all the preexisting correlations between the turbulent fluctuations in the solar wind. This results in suppressing the Kolmogorov inertial range and generating locally random-like fluctuations that have a scaling of $\sim f^{-1}$ over a broad range of scales. Those scales would play the same role as the energy-containing scales in solar wind turbulence. The absence of the inertial range scale in our observations can be explained by the fact that the newly generated fluctuations behind the shock do not have "enough time" to interact sufficiently with each other to reach a fully developed turbulence state, hence the direct transition from the "energy-containing" range that has $\sim f^{-1}$ scaling into the subion range with a scaling of $\sim f^{-2.6}$. In this scenario, turbulence may reach a fully developed state and the Kolmogorov 5/3 spectrum may be observed, but only far away from the shock (e.g., toward the flanks). However, a fundamental question remains open. How are the power-law spectra observed in the sub-ion range created in the absence of an inertial range? We note that existing theoretical models of kinetic turbulence in the 
Quasi-Perpendicular Shock 2007/04/28 13:30-17:00

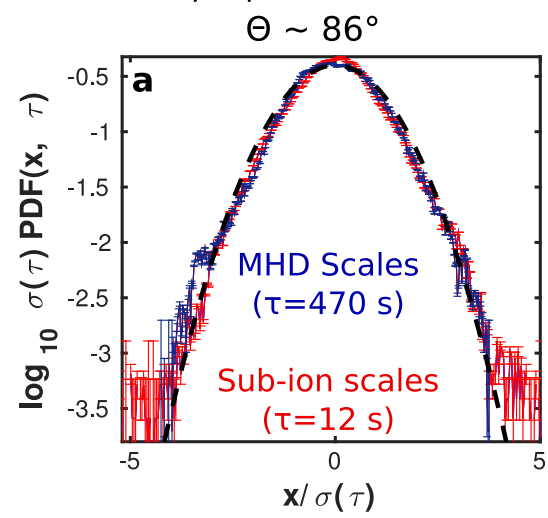

Quasi-Perpendicular Shock 2005/03/17 06:00-08:20 $\Theta \sim 60^{\circ}$

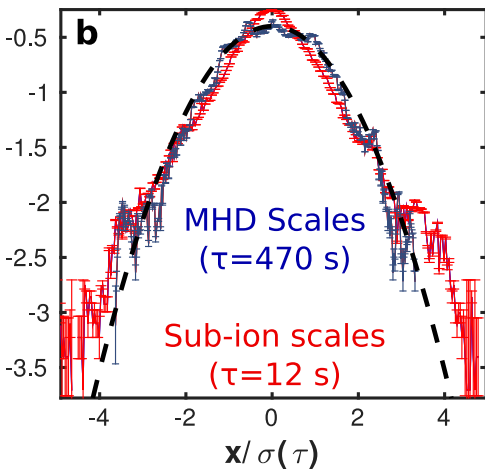

Quasi-Parallel Shock 2005/03/17 02:05-04:15

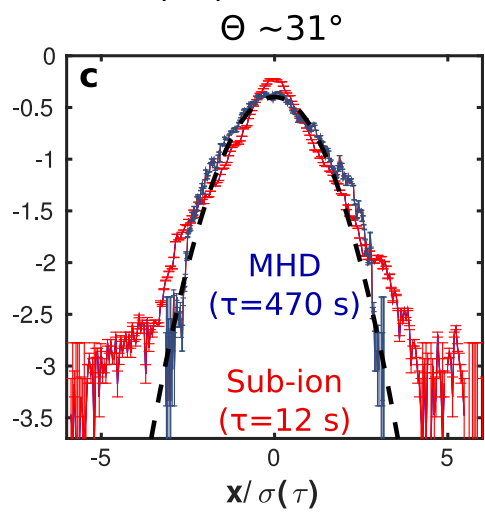

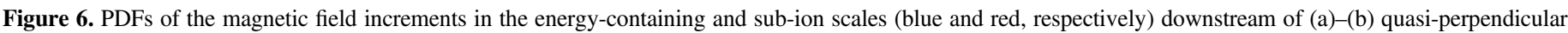

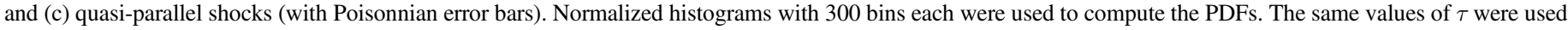

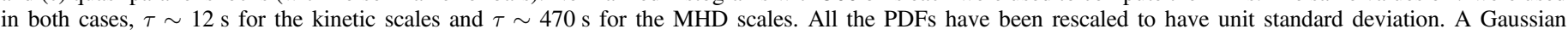
distribution (black dashed curve) is shown for comparison.
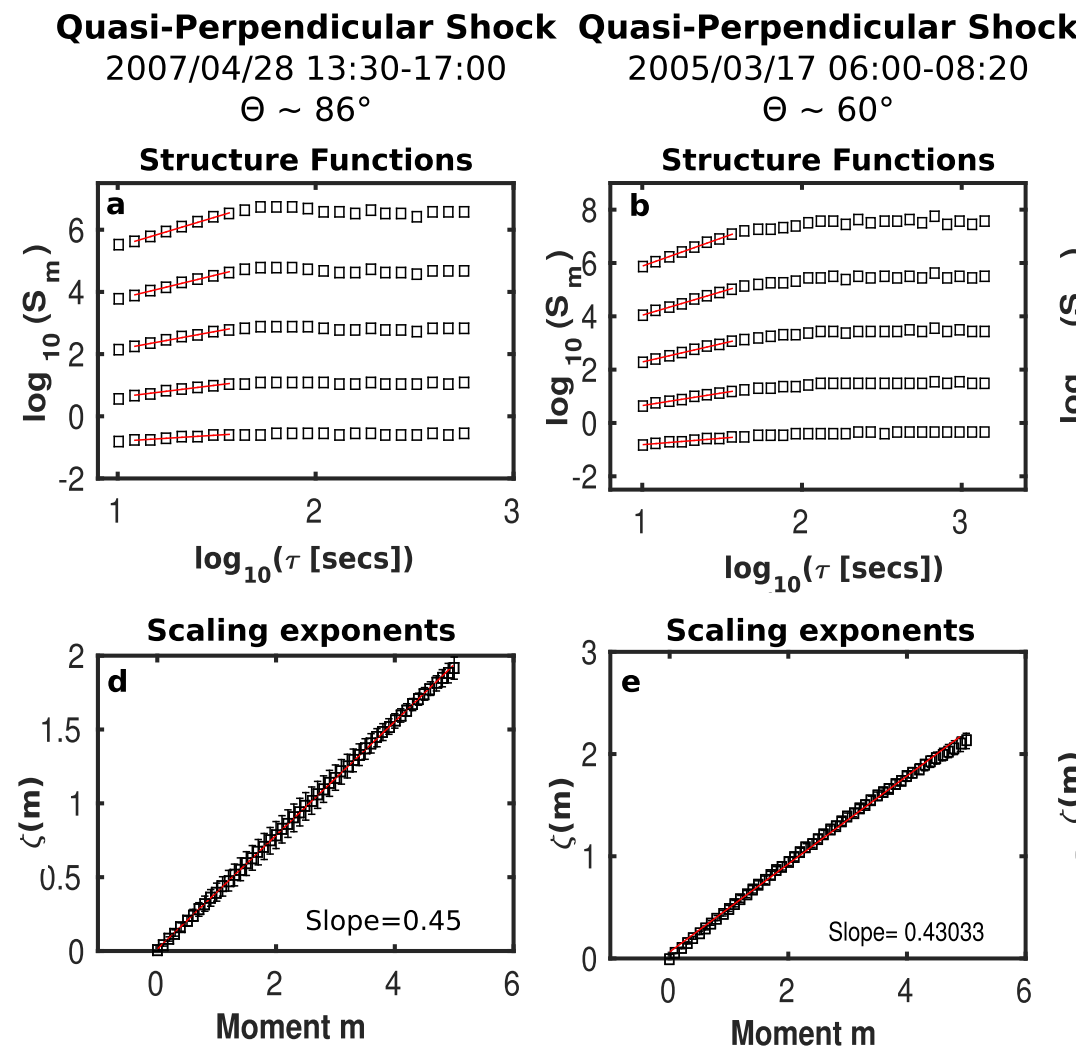

Quasi-Parallel Shock 2005/03/17 02:05-04:15

$\Theta \sim 31^{\circ}$
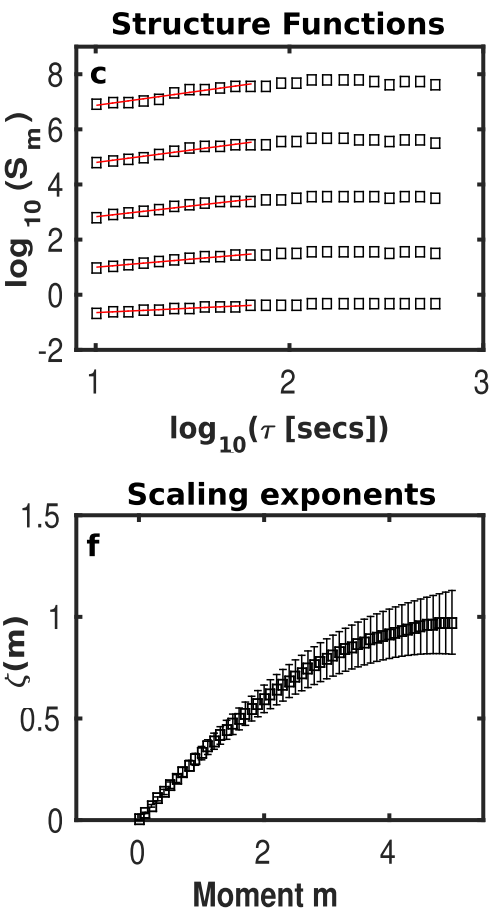

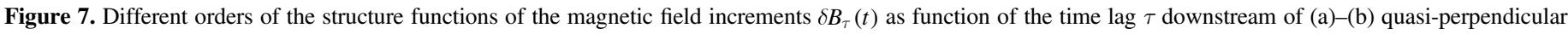
and (c) quasi-parallel shocks. Panels (d)-(f) represent the corresponding scaling exponent $\zeta(m)$.

solar wind suggest that turbulence at sub-ion scales may result from a decoupling between the dynamics of Alfvénic fluctuations and the rest of the MHD fluctuations that would carry the cascade into kinetic scales (Schekochihin et al. 2009). Hence, if the MHD scales are not dominated by Alfvénic fluctuations as observed here, it is not clear as to how turbulence is generated at kinetic scales. Is it generated by local plasma instabilities occurring near the ion scale as proposed in Sahraoui et al. (2006)? In this case, would the $f^{-1}$ spectrum observed at larger scales result from an inverse cascade as observed in hydrodynamic turbulence (Paret \& Tabeling 1997;
Chertkov et al. 2007)? Another observation reported here that requires further investigation is the nature of the turbulence observed behind the quasi-perpendicular shock. At sub-ion scales, turbulence was found to have a monofractal behavior as in the solar wind (Kiyani et al. 2009a). However, the tails of the PDFs are clearly less pronounced in our observations and the PDF of Figure 6(a) looked close to a Gaussian rather than a heavy-tailed PDF. This result recalls recent numerical observations of weak turbulence in electron-MHD (Meyrand et al. 2015). This similarity and the questions raised above require further investigation in the future. 
The authors thank NASA's Planetary Data System (PDS) for the CASSINI-CAPS data set availability (Waite, J.H., Furman, J.D., CASSINI ORBITER SAT/SW CAPS DERIVED ION MOMENTS V1.0, CO-S/SW-CAPS-5-DDR-ION-MOMENTSV1.0, NASA Planetary Data System, 2013). The authors are also indebted to the program "Soleil Héliosphère et Magnétosphères" of CNES, the French Space Administration for the financial support on CASSINI. Data analysis was done with the AMDA science analysis system provided by the Centre de Données de la Physique des Plasmas (IRAP, Université Paul Sabatier, Toulouse) supported by CNRS and CNES. L.H. thanks G. Belmont for many fruitful discussions.

\section{REFERENCES}

Alexandrova, O., Lacombe, C., \& Mangeney, A. 2008, AnGeo, 26, 3585 Bagenal, F. 2007, JASTP, 69, 387

Bavassano, B., Dobrowolny, M., Mariani, F., \& Ness, N. F. 1982, JGRA, 87,3617

Cattaneo, M. B. B., Moreno, G., Russo, G., \& Richardson, J. D. 2000, JGRA, 105,23141

Chertkov, M., Connaughton, C., Kolokolov, I., \& Lebedev, V. 2007, PhRvL, 99, 084501

Chust, T., Roux, A., Kurth, W., et al. 2005, P\&SS, 53, 395

Czaykowska, A., Bauer, T. M., Treumann, R. A., \& Baumjohann, W. 2001, AnGeo, 19, 275

Dougherty, M. K., Kellock, S., Southwood, D. J., et al. 2004, SSRv, 114, 331

Erds, G., \& Balogh, A. 1996, JGRA, 101, 1

Gary, S. P., \& Smith, C. W. 2009, JGRA, 114, A12105

Gary, S. P., \& Winske, D. 1992, JGRA, 97, 3103

Goldstein, M. L., Roberts, D. A., \& Fitch, C. A. 1994, JGRA, 99, 11519

Hamilton, K., Smith, C. W., Vasquez, B. J., \& Leamon, R. J. 2008, JGR, 113 , A01106

Hollweg, J. V. 1999, JGR, 104, 14811

Howes, G. G. 2009, NPGeo, 16, 219

Howes, G. G., Bale, S. D., Klein, K. G., et al. 2012, ApJL, 753, L19

Howes, G. G., Cowley, S. C., Dorland, W., et al. 2006, ApJ, 651, 590

Huang, S. Y., Sahraoui, F., Deng, X. H., et al. 2014, ApJL, 789, L28

Iroshnikov, P. S. 1964, SvA, 7, 566

Ito, A., Hirose, A., Mahajan, S. M., \& Ohsaki, S. 2004, PhPl, 11, 5643

Karimabadi, H., Roytershteyn, V., Vu, H. X., et al. 2014, PhP1, 21, 062308

Kaufmann, R. L., Horng, J.-T., \& Wolfe, A. 1970, JGR, 75, 4666

Kivelson, M. G., Bagenal, F., Kurth, W. S., et al. 2004, in The Planet, Satellites, and Magnetosphere, ed. F. Bagenal, T. E. Dowling, \& W. B. McKinnon (Cambridge: Cambridge Univ. Press), 537

Kiyani, K. H., Chapman, S. C., Khotyaintsev, Yu. V., Dunlop, M. W., \& Sahraoui, F. 2009a, PhRvL, 103, 075006

Kiyani, K. H., Chapman, S. C., Sahraoui, F., et al. 2013, ApJ, 763, 10
Kiyani, K. H., Chapman, S. C., \& Watkins, N. W. 2009b, PhRvE, 79, 036109 Klein, K. G., Howes, G. G., TenBarge, J. M., et al. 2012, ApJ, 755, 159

Kraichnan, R. 1965, PhFl, 8, 1385

Leamon, R. J., Matthaeus, W. H., Smith, C. W., \& Wong, H. K. 1998a, ApJL, 507, L181

Leamon, R. J., Smith, C. W., Ness, N. F., Matthaeus, W. H., \& Wong, H. K. 1998b, JGRA, 103, 4775

Leamon, R. J., Smith, C. W., Ness, N. F., Matthaeus, W. H., \& Wong, H. K. 1999, JGRA, 104, 22331

Lewis, G. R., Andr, N., Arridge, C. S., et al. 2008, P\&SS, 56, 901

Mangeney, A., Lacombe, C., Maksimovic, M. S.-A. A., et al. 2006, AnGeo, 24,3507

Masters, A., Schwartz, S. J., Henley, E. M., et al. 2011, JGRA, 116, A10107

Masters, A. 2013, NatPh, 9, 164

Meyrand, R., Kiyani, K. H., \& Galtier, S. 2015, JFM, 770, R1

Paret, J., \& Tabeling, P. 1997, PhRvL, 79, 4162

Podesta, J. J., \& TenBarge, J. M. 2012, JGRA, 117, A10106

Romanelli, N., Modolo, R., Dubinin, E., et al. 2014, JGRA, 119, 9992

Rönnmark, K. 1982, WHAMP-Waves in Homogeneous, Anisotropic, Multicomponent Plasmas, Tech. Rep. 179 (Kiruna Geophysical Institute)

Russell, C. T., Lepping, R. P., \& Smith, C. W. 1990, JGR, 95, 2273

Sahraoui, F., Belmont, G., \& Goldstein, M. L. 2012, ApJ, 748, 100

Sahraoui, F., Belmont, G., Rezeau, L., \& Cornilleau-Wehrlin, N. 2006, PhRvL, 96, 075002

Sahraoui, F., Goldstein, M. L., Belmont, G., Canu, P., \& Rezeau, L. 2010, PhRvL, 105, 131101

Sahraoui, F., Goldstein, M. L., \& Khotyaintse, Y. V. 2009, PhRvL, 102, 231102

Sahraoui, F., Huang, S. Y., Belmont, G., et al. 2013, ApJ, 777, 15

Sahraoui, F., Pinçon, J. L., Belmont, G., et al. 2003, JGRA, 108, 1335

Salem, C. S., Howes, G. G., Sundkvist, D., et al. 2012, ApJL, 745, L9

Saur, J., Neubauer, F. M., Connerney, J. E. P., Zarka, P., \& Kivelson, M. G. 2004, in The Planet, Satellites, and Magnetosphere, ed. F. Bagenal, T. E. Dowling \& W. B. McKinnon (Cambridge: Cambridge Univ. Press), 537

Schekochihin, A. A., Cowley, S. C., Dorland, W., et al. 2009, ApJS, 182, 310

Song, P., Russell, C. T., \& Gary, S. P. 1994, JGRA, 99, 6011

Southwood, D. J., \& Kivelson, M. G. 1993, JGRA, 98, 9181

Sundkvist, D., Retinò, A., Vaivads, A., \& Bale, S. D. 2007, PhRvL, 99, 025004

Tao, C., Sahraoui, F., Fontaine, D., et al. 2015, JGRA, 120, 2477

Thomsen, M. F., Reisenfeld, D. B., Delapp, D. M., et al. 2010, JGRA, 115,2156

Velli, M., Grappin, R., \& Mangeney, A. 1989, PhRvL, 63, 1807

Violante, L., Cattaneo, M. B. B., Moreno, G., \& Richardson, J. D. 1995, JGRA, 100, 12047

von Papen, M., Saur, J., \& Alexandrova, O. 2014, JGRA, 119, 2797

Went, D. R., Hospodarsky, G. B., Masters, A., Hansen, K. C., \& Dougherty, M. K. 2011, JGRA, 116, a07202

Yordanova, E., Vaivads, A., André, M., Buchert, S. C., \& Vörös, Z. 2008, PhRvL, 100, 205003

Young, D. T., Berthelier, J. J., Blanc, M., et al. 2004, SSRv, 114, 1 